Supporting Information

\title{
Facile Synthesis of $\mathrm{MnFe}_{2} \mathrm{O}_{4}$ Hollow Sphere-Reduced Graphene
} Oxide Nanocomposites as Electrode Materials for All-Solid-State Flexible High-Performance Asymmetric Supercapacitors

Priyanka Makkar, and Narendra Nath Ghosh*

Nano-materials Lab, Department of Chemistry, Birla Institute of Technology and Science, Pilani K K Birla Goa Campus, Goa-403726, India.

*Corresponding author. Tel. /fax: +91 832 2580318/25570339.

*E-mail address: naren70@yahoo.com (N. N. Ghosh) 
Chemicals. Manganese chloride tetrahydrate $\left(\mathrm{MnCl}_{2} .4 \mathrm{H}_{2} \mathrm{O}\right)$, iron chloride hexahydrate $\left(\mathrm{FeCl}_{3} .6 \mathrm{H}_{2} \mathrm{O}\right)$, and polyethylene glycol (PEG) were purchased from Molychem. Sodium acetate (NaAc), ethanol and acetone were procured from Fischer Scientific. Ethylene glycol (EG) was purchased from Merk, India.

Characterization and Instrumentation. In the present study, the synthesized pure $\mathrm{MnFe}_{2} \mathrm{O}_{4}$ and the nanocomposites were characterized by room temperature powder X-ray diffraction (XRD) patterns of the synthesized materials were recorded using a powder X-ray diffraction (XRD) (Mini Flex II, Rigaku) with $\mathrm{Cu} \mathrm{K}_{\alpha}(\lambda=0.15405 \mathrm{~nm})$ radiation at a scanning speed of $3{ }^{\circ} \mathrm{min}^{-1}$, Thermogravimetric analysis (TGA) of the samples were performed in air atmosphere by using DTG-60 SHIMADZU), Fourier Transform Infrared spectra (FT-IR) were recorded in $\mathrm{KBr}$ by using spectrophotometer (IR Affinity-1, Shimadzu). Raman spectra were recorded on a Horiba via Raman microscope with a 633 nm laser excitation, Field emission scanning electron microscopy (FESEM) images of samples were obtained using Quanta 250 FEG (FEI), and Energy-dispersive X-ray spectra (EDS) of the synthesized material was recorded using an EDAX ELEMENT electron microscope. IVIUMSTAT $(10 \mathrm{~V} / 5 \mathrm{~A} / 8 \mathrm{MHz})$ workstation was used to perform the electrochemical studies.

Electrochemical Measurements. First, a viscous paste was prepared by mixing $80 \mathrm{wt} \%$ of active material, $10 \mathrm{wt} \%$ polyvinylidene difluoride in $\mathrm{N}$-methyl-2-pyrrolidinone and $10 \mathrm{wt} \%$ acetylene black. The working electrode was fabricated by loading $\sim 2 \mathrm{mg}$ of this paste on a Ni foam with the dimension of $(1.5 \mathrm{~cm} \times 1.5 \mathrm{~cm} \times 0.2 \mathrm{~mm})$ and then dried it at $80{ }^{\circ} \mathrm{C}$ for $12 \mathrm{~h}$ under vacuum. In the three-electrode system, a $\mathrm{Hg} / \mathrm{HgO}$ electrode and a $\mathrm{Pt}$ wire were used as the reference electrode and the counter electrode, respectively. Electrochemical measurements were conducted using two electrolytes (i) $3 \mathrm{M}$ $\mathrm{KOH}$ aqueous solution, and (ii) an aqueous mixture of $3 \mathrm{M} \mathrm{KOH}$ and $0.1 \mathrm{M} \mathrm{K} 4[\mathrm{Fe}(\mathrm{CN}) 6]$. 
Asymmetric System. The voltammetric charges (Q) were calculated based on the following equations:

$$
\mathrm{Q}=\mathrm{C}_{\text {single }} \times \Delta \mathrm{V} \times \mathrm{m}
$$

where $\mathrm{m}$ is the mass of the electrode $(\mathrm{g}), \Delta \mathrm{V}$ is the potential window $(\mathrm{V})$, and $\mathrm{C}_{\text {single }}$ is the specific capacitance $\left(\mathrm{F} \mathrm{g}^{-1}\right)$ of each electrode measured in three-electrode setup (calculated from cyclic voltammograms at a scan rate of $\left.10 \mathrm{mV} \mathrm{s}^{-1}\right)$.

Balancing of charge is carried out by substituting above equation for both anode and cathode considering their charge/mass ratio as:

$$
\frac{q_{+}}{q_{-}}=\frac{m_{+}}{m_{-}}=\frac{C_{s p-} \times \Delta V^{-}}{C_{s p+} \times \Delta V^{+}}
$$

The CV and GCD measurements were carried out using a galvanostat-potentiostat. For the threeelectrode setup, all the CV measurements were conducted in the working potential window between 0 $0.55 \mathrm{~V}$ by using various scan rates between 10 to $100 \mathrm{mV} \mathrm{s}^{-1}$. For the symmetric cell $\left(80 \mathrm{MnFe}_{2} \mathrm{O}_{4}\right.$ $20 \mathrm{rGO} / / 80 \mathrm{MnFe}_{2} \mathrm{O}_{4}-20 \mathrm{rGO}$ ), the working potential window was $0-1 \mathrm{~V}$ and for the asymmetric cell setup $\left(80 \mathrm{MnFe}_{2} \mathrm{O}_{4}-20 \mathrm{rGO} / / \mathrm{rGO}\right)$, the potential window was 0-1.5 V. The GCD measurements were performed at different current densities varying from 3 to $12 \mathrm{~A} \mathrm{~g}^{-1}$ in case of symmetric cell whereas, for asymmetric cell, the measurements were performed at current densities varying from 1 to $10 \mathrm{~A} \mathrm{~g}^{-1}$. The equations used to determine specific capacitance $\left(\mathrm{C}_{S}\right)$, power density and energy density are mentioned in the supporting information (equation S3-S11).

Electrochemical Impedance Spectroscopy (EIS) measurements were performed in the frequency range of $0.01-10000 \mathrm{~Hz}$ at open circuit potential with an alternating current amplitude of $0.01 \mathrm{~V}$.

\section{Equations:}


The values of $\mathrm{C}_{\mathrm{S}}$, energy density $(\mathrm{E})$, and power density $(\mathrm{P})$ for three-electrode setup were determined by using the following equations:

$$
\begin{aligned}
C_{s} & =\frac{i \Delta t}{m \Delta V} \\
E & =\frac{C_{s} \times(\Delta V)^{2}}{2} \\
P & =\frac{E}{\Delta t}
\end{aligned}
$$

where $\mathrm{C}_{\mathrm{s}}$ is the specific capacitance based on the mass of the electroactive material $\left(\mathrm{F} \mathrm{g}^{-1}\right)$, i (A) represents the charge or discharge current, $\Delta \mathrm{t}(\mathrm{s})$ is the discharge time, $\mathrm{m}(\mathrm{g})$ represents the mass of supercapacitive material and $\Delta \mathrm{V}$ is the applied potential window, $\mathrm{E}$ is the average energy density (Wh $\left.\mathrm{kg}^{-1}\right), \mathrm{P}$ is the power density $\left(\mathrm{W} \mathrm{kg}^{-1}\right)$, and $\Delta \mathrm{t}$ is the discharge time (s).

\section{Two-electrode Symmetric Cell}

The values of $\mathrm{C}_{\mathrm{S}}$, energy density $(\mathrm{E})$, and power density $(\mathrm{P})$ for two-electrode symmetric setup were determined by using the following equations:

$$
\begin{aligned}
& C_{S}=\frac{4 i \Delta t}{m \Delta V} \\
& E=\frac{1}{8} C_{S}(\Delta V)^{2} \\
& P=\frac{E}{\Delta t}
\end{aligned}
$$

where $\mathrm{C}_{\mathrm{S}}$ is the specific capacitance based on the mass of the electroactive material $\left(\mathrm{F} \mathrm{g}^{-1}\right), \mathrm{i}(\mathrm{A})$ represents the charge or discharge current, $\Delta \mathrm{t}(\mathrm{s})$ is the discharge time, $\mathrm{m}(\mathrm{g})$ represents the mass of 
supercapacitive material and $\Delta \mathrm{V}$ is the applied potential window, $\mathrm{E}$ is the average energy density (Wh $\left.\mathrm{kg}^{-1}\right), \mathrm{P}$ is the power density $\left(\mathrm{W} \mathrm{kg}^{-1}\right)$, and $\Delta \mathrm{t}$ is the discharge time (s).

\section{Two-electrode Asymmetric Cell}

The values of $\mathrm{C}_{\mathrm{S}}$, energy density (E), and power density (P) for two-electrode asymmetric setup were determined by using the following equations:

$$
\begin{gathered}
C_{S}=\frac{i \Delta t}{m \Delta V} \\
E=\frac{C_{s} \times(\Delta V)^{2}}{2} \\
P=\frac{E}{\Delta t}
\end{gathered}
$$

where $\mathrm{C}_{\mathrm{S}}$ is the specific capacitance based on the mass of the electroactive material $\left(\mathrm{F} \mathrm{g} \mathrm{g}^{-1}\right), \mathrm{i}(\mathrm{A})$ represents the charge or discharge current, $\Delta \mathrm{t}(\mathrm{s})$ is the discharge time, $\mathrm{m}(\mathrm{g})$ represents the mass of supercapacitive material and $\Delta \mathrm{V}$ is the applied potential window, $\mathrm{E}$ is the average energy density (Wh $\left.\mathrm{kg}^{-1}\right), \mathrm{P}$ is the power density $\left(\mathrm{W} \mathrm{kg}^{-1}\right)$, and $\Delta \mathrm{t}$ is the discharge time (s).

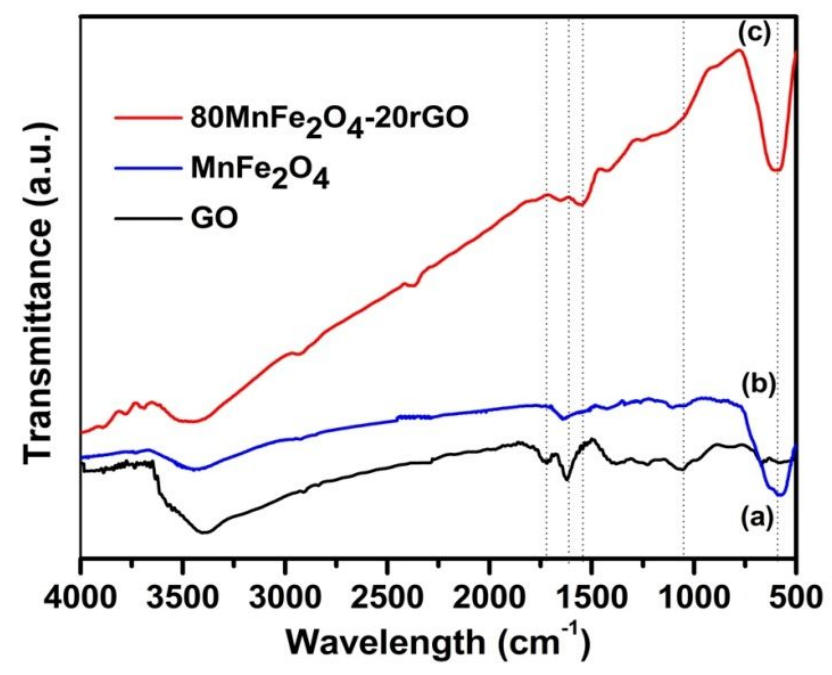

Figure S1. FT-IR spectra of (a) GO, (b) $\mathrm{MnFe}_{2} \mathrm{O}_{4}$, and (c) $80 \mathrm{MnFe}_{2} \mathrm{O}_{4}-20 \mathrm{rGO}$. 


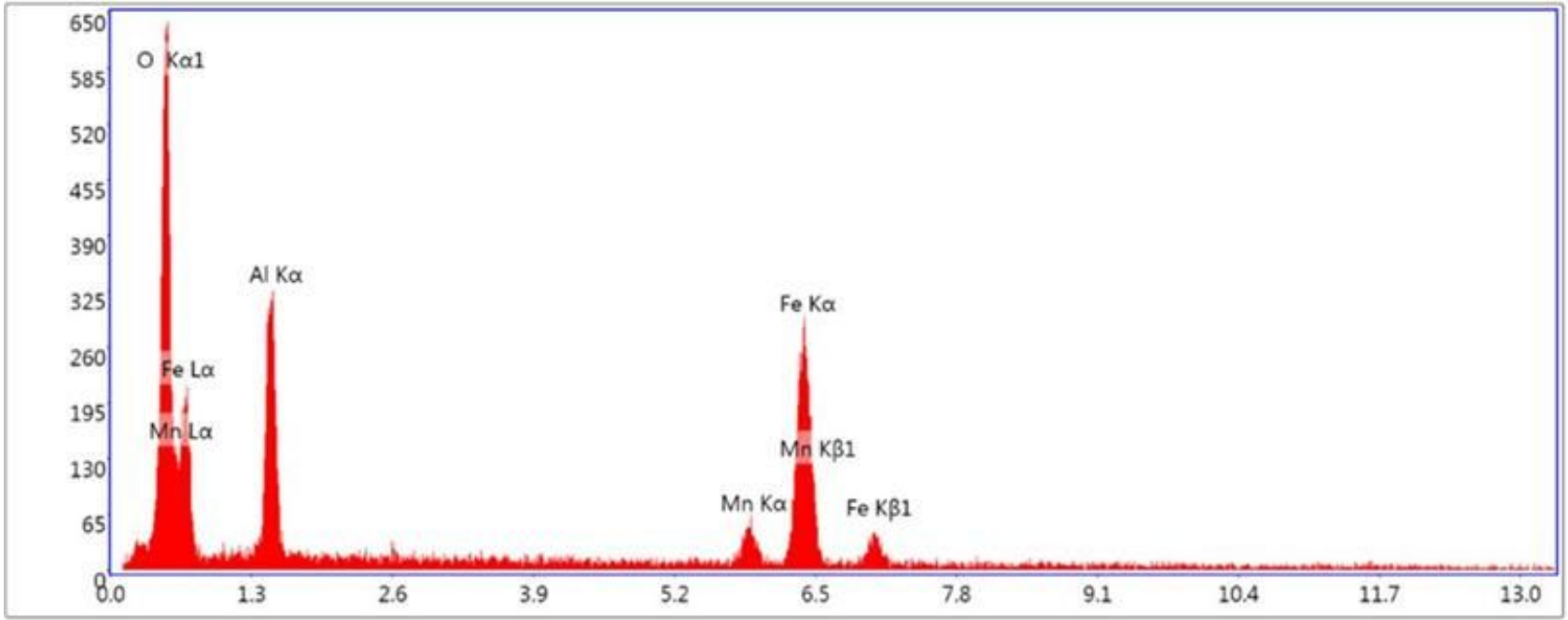

Figure S2. EDS analysis of $\mathrm{MnFe}_{2} \mathrm{O}_{4}$ hollow spheres.

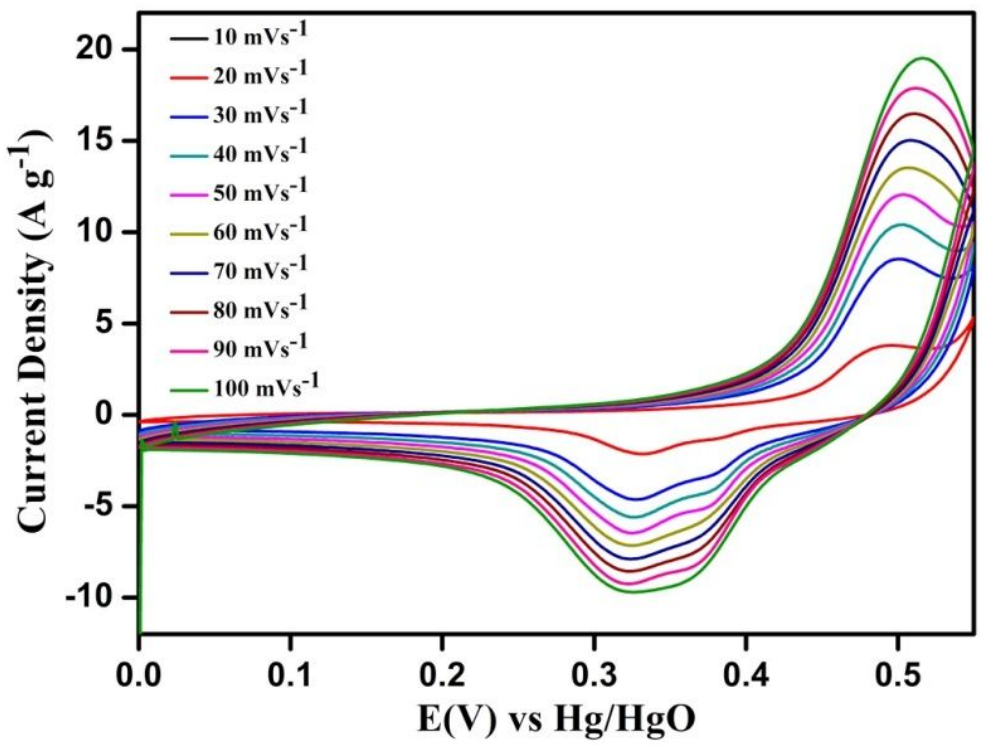

Figure S3. CV curves of pure $\mathrm{MnFe}_{2} \mathrm{O}_{4}$ electrode in $3 \mathrm{M} \mathrm{KOH}$ at different scan rates ranging from 10$100 \mathrm{mV} \mathrm{s}^{-1}$. 


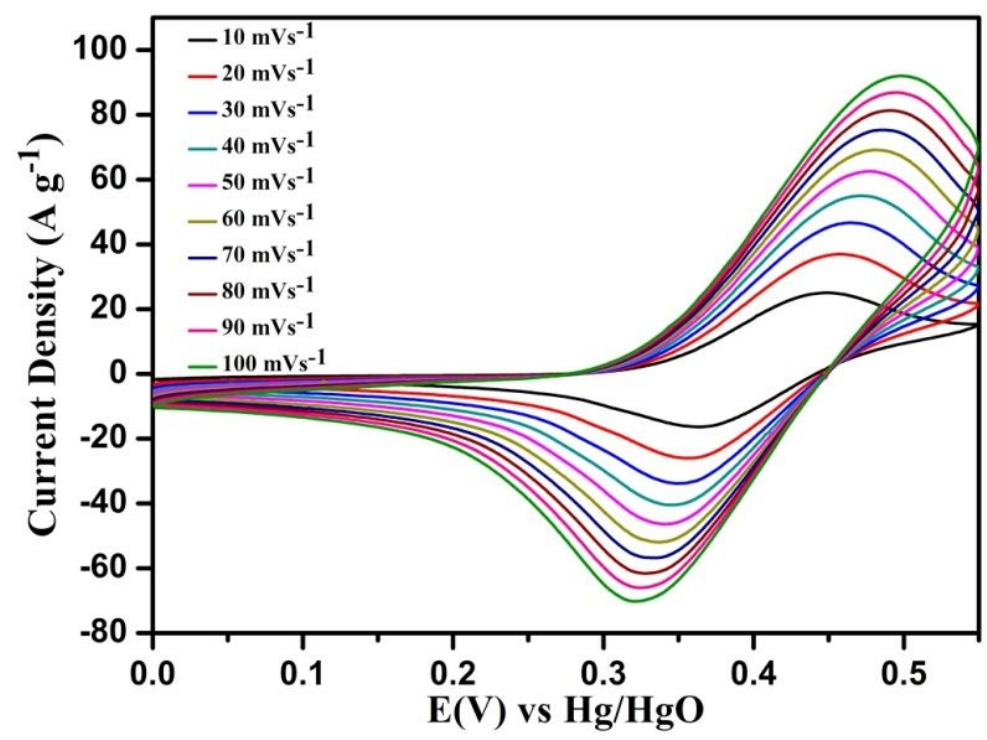

Figure S4. $\mathrm{CV}$ curves of pure $\mathrm{MnFe}_{2} \mathrm{O}_{4}$ electrode in $3 \mathrm{M} \mathrm{KOH}+0.1 \mathrm{M} \mathrm{K}\left[\mathrm{Fe}(\mathrm{CN})_{6}\right]$ at different scan rates ranging from $10-100 \mathrm{mV} \mathrm{s}^{-1}$.

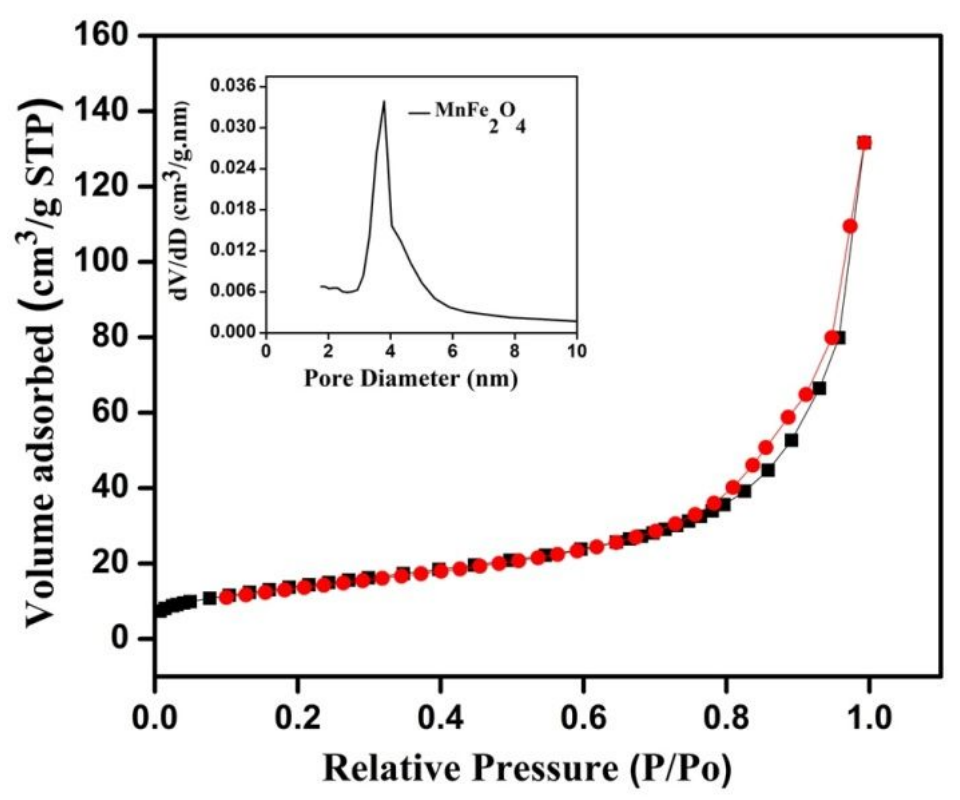

Figure S5. $\mathrm{N}_{2}$ adsorption-desorption isotherms and (inset) pore size distribution of synthesized $\mathrm{MnFe}_{2} \mathrm{O}_{4}$. 


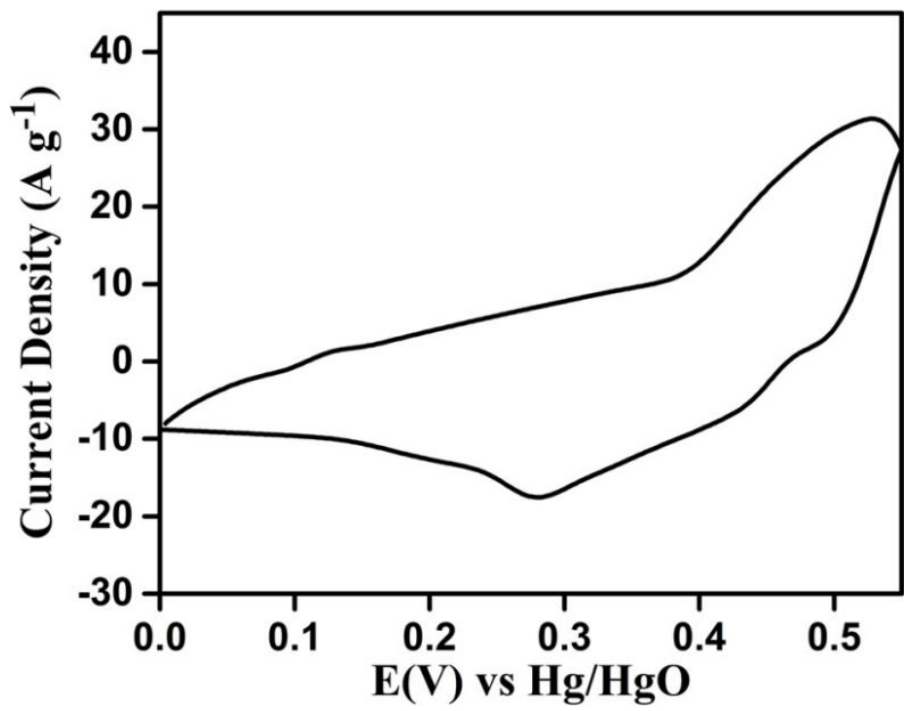

Figure S6. CV curve of pure rGO electrode in $3 \mathrm{M} \mathrm{KOH}+0.1 \mathrm{M} \mathrm{K}_{4}\left[\mathrm{Fe}(\mathrm{CN})_{6}\right]$.
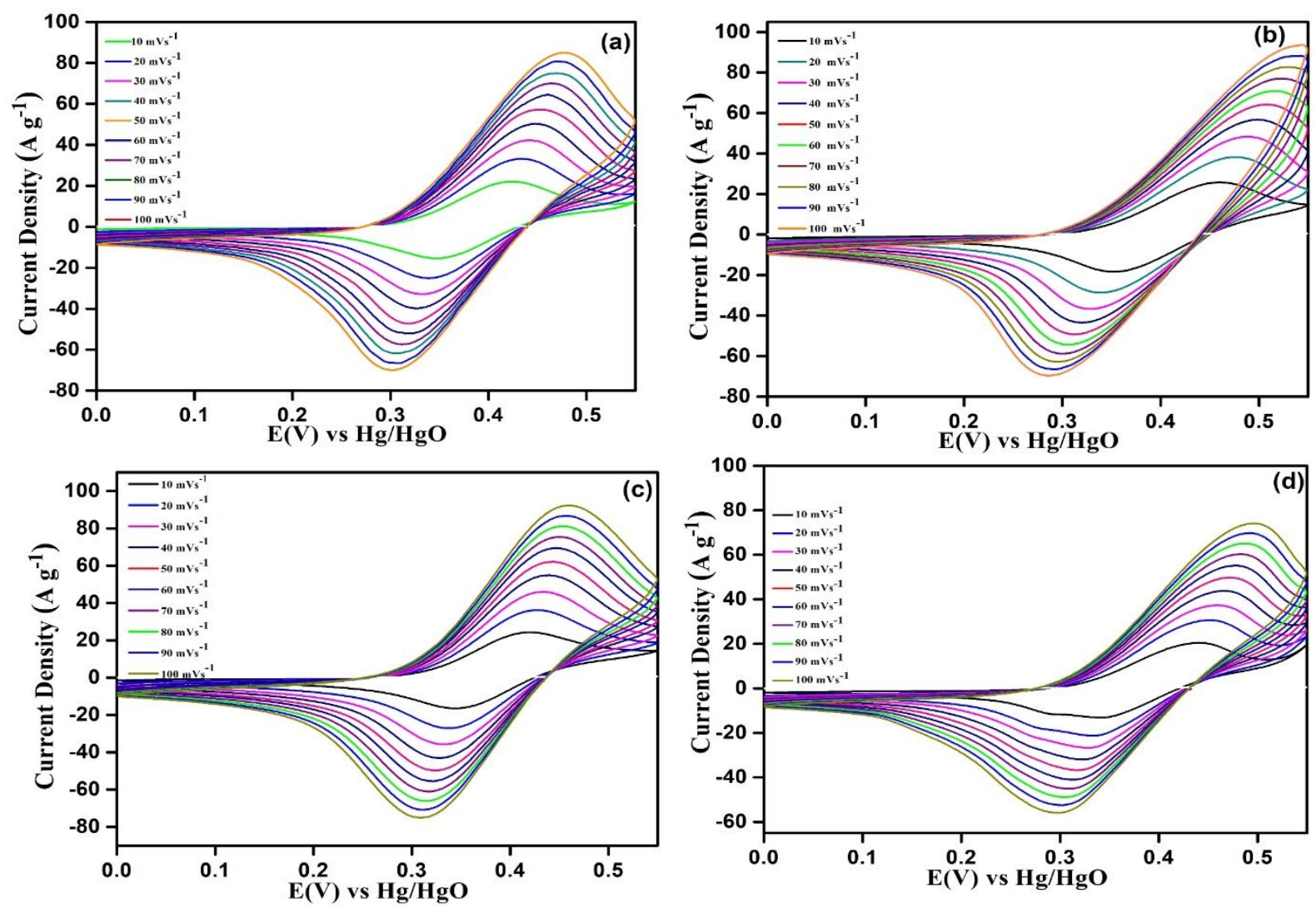

Figure S7. CV curves of (a) $95 \mathrm{MnFe}_{2} \mathrm{O}_{4}-5 \mathrm{rGO}$, (b) $90 \mathrm{MnFe}_{2} \mathrm{O}_{4}-10 \mathrm{rGO}$, (c) $70 \mathrm{MnFe}_{2} \mathrm{O}_{4}-30 \mathrm{rGO}$, and

(d) $60 \mathrm{MnFe}_{2} \mathrm{O}_{4}-40 \mathrm{rGO}$ electrode in $3 \mathrm{M} \mathrm{KOH}+0.1 \mathrm{M} \mathrm{K}_{4}\left[\mathrm{Fe}(\mathrm{CN})_{6}\right]$ at different scan rate ranging from $10-100 \mathrm{mV} \mathrm{s}^{-1}$. 

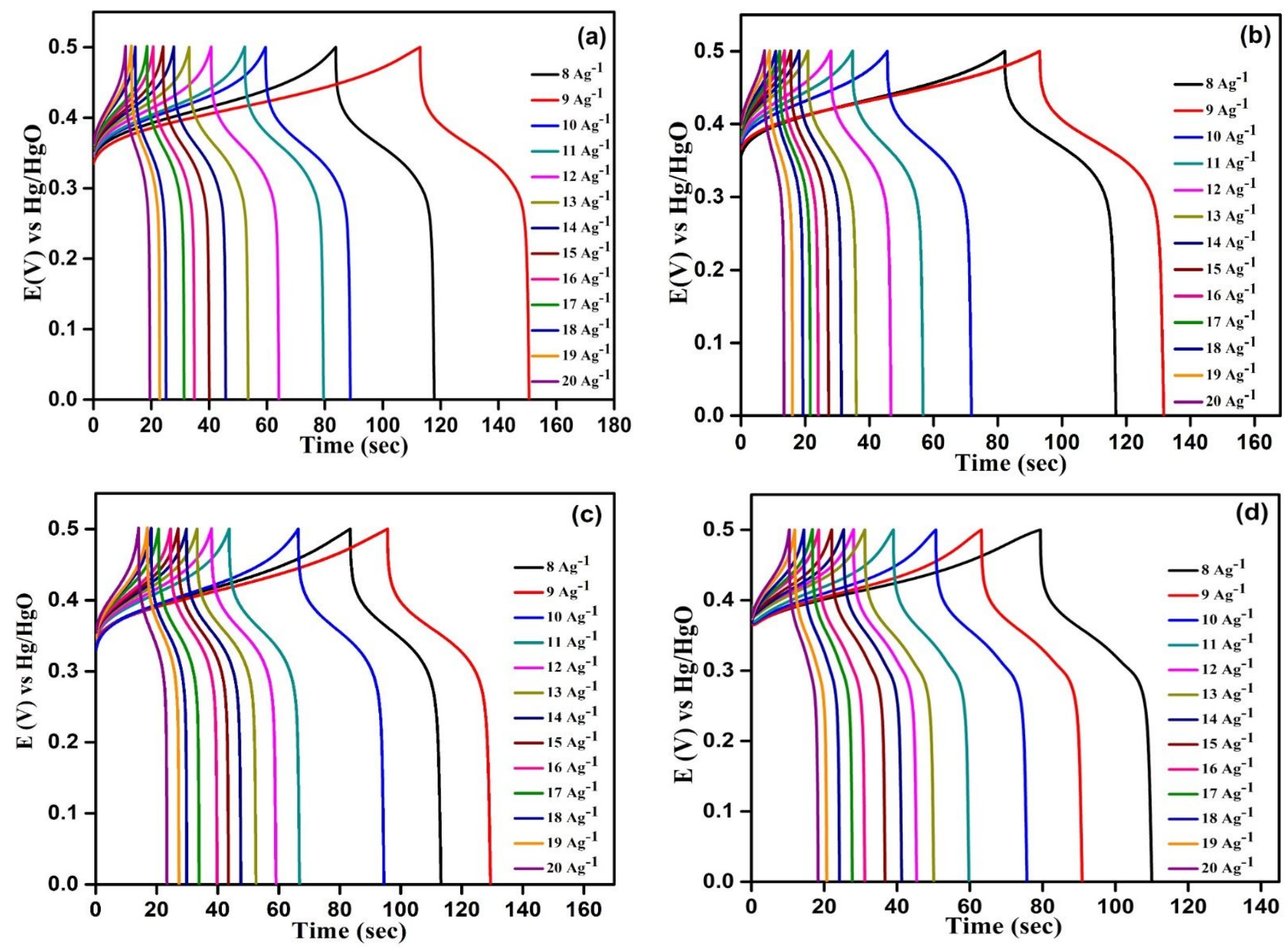

Figure S8. GCD curves of (a) $95 \mathrm{MnFe}_{2} \mathrm{O}_{4}-5 \mathrm{rGO}$, (b) $90 \mathrm{MnFe}_{2} \mathrm{O}_{4}-10 \mathrm{rGO}$, (c) $70 \mathrm{MnFe}_{2} \mathrm{O}_{4}-30 \mathrm{rGO}$, and

(d) $60 \mathrm{MnFe}_{2} \mathrm{O}_{4}-40 \mathrm{rGO}$ electrode at different current densities $\left(8-20 \mathrm{~A} \mathrm{~g}^{-1}\right)$ in $3 \mathrm{M} \mathrm{KOH}+0.1 \mathrm{M}$ $\mathrm{K}_{4}\left[\mathrm{Fe}(\mathrm{CN})_{6}\right]$ 
Table S1. Comparison of electrochemical performance of Manganese ferrite and graphene-based nanocomposites with varying rGO content in three-electrode supercapacitors.

\begin{tabular}{|c|c|c|c|c|c|}
\hline S.No & Material & Electrolyte & $\begin{array}{c}\text { Specific } \\
\text { capacitance } \\
\left(\mathrm{F} \mathrm{g} \mathrm{g}^{-1}\right)\end{array}$ & $\begin{array}{c}\text { Power Density } \\
\left(W k^{-1}\right)\end{array}$ & $\begin{array}{c}\text { Energy Density } \\
\left(\mathrm{Wh} \mathrm{kg}^{-1}\right)\end{array}$ \\
\hline 1) & $95 \mathrm{MnFe}_{2} \mathrm{O}_{4}-5 \mathrm{rGO}$ & $\begin{array}{c}3 \mathrm{M} \mathrm{KOH}+ \\
0.1 \mathrm{M} \\
\mathrm{K}_{4}\left[\mathrm{Fe}(\mathrm{CN})_{6}\right]\end{array}$ & $\begin{array}{c}617.6 \\
\left(8 \mathrm{~A} \mathrm{~g}^{-1}\right)\end{array}$ & 2000 & 21.44 \\
\hline 2) & $90 \mathrm{MnFe}_{2} \mathrm{O}_{4}-10 \mathrm{rGO}$ & $\begin{array}{c}3 \mathrm{M} \mathrm{KOH}+ \\
0.1 \mathrm{M} \\
\mathrm{K}_{4}\left[\mathrm{Fe}(\mathrm{CN})_{6}\right]\end{array}$ & $\begin{array}{c}624 \\
\left(8 \mathrm{~A} \mathrm{~g}^{-1}\right)\end{array}$ & 2000 & 21.66 \\
\hline 3) & $80 \mathrm{MnFe}_{2} \mathrm{O}_{4}-20 \mathrm{rGO}$ & $\begin{array}{c}3 \mathrm{M} \mathrm{KOH}+ \\
0.1 \mathrm{M} \\
\mathrm{K}_{4}\left[\mathrm{Fe}(\mathrm{CN})_{6}\right]\end{array}$ & $\begin{array}{c}768 \\
\left(8 \mathrm{~A} \mathrm{~g}^{-1}\right)\end{array}$ & 2000 & 26.26 \\
\hline 4) & $70 \mathrm{MnFe}_{2} \mathrm{O}_{4}-30 \mathrm{rGO}$ & $\begin{array}{c}3 \mathrm{M} \mathrm{KOH}+ \\
0.1 \mathrm{M} \\
\mathrm{K}_{4}\left[\mathrm{Fe}(\mathrm{CN})_{6}\right]\end{array}$ & $\begin{array}{c}556.8 \\
\left(8 \mathrm{~A} \mathrm{~g}^{-1}\right)\end{array}$ & 2000 & 19.66 \\
\hline 5) & $60 \mathrm{MnFe}_{2} \mathrm{O}_{4}-40 \mathrm{rGO}$ & $\begin{array}{c}3 \mathrm{M} \mathrm{KOH}+ \\
0.1 \mathrm{M} \\
\mathrm{K}_{4}\left[\mathrm{Fe}(\mathrm{CN})_{6}\right]\end{array}$ & 496 & 2000 & 17.22 \\
\hline
\end{tabular}




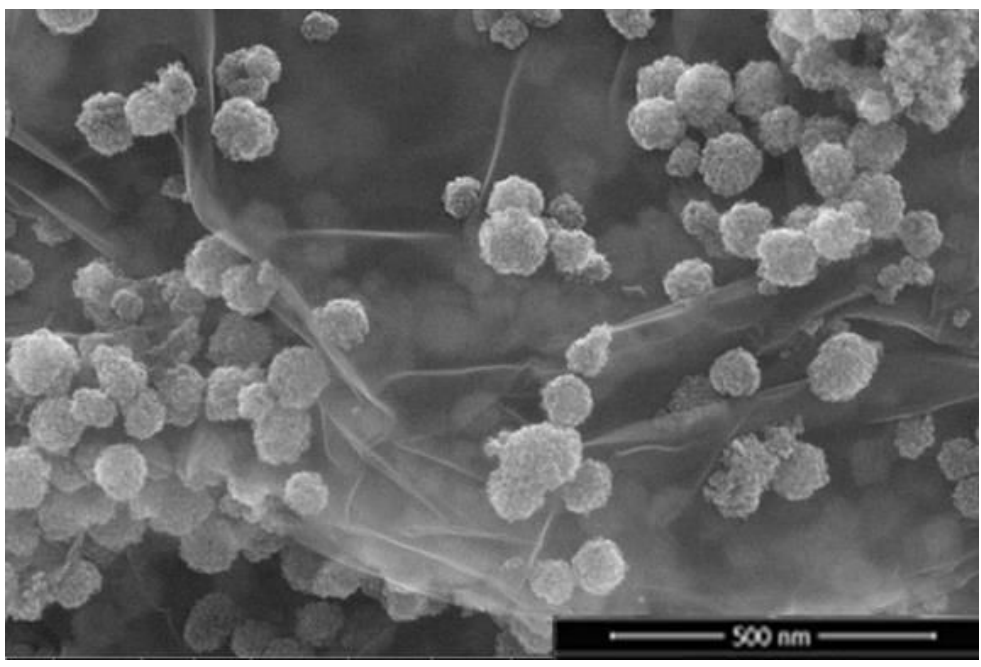

Figure S9. FESEM micrograph of $70 \mathrm{MnFe}_{2} \mathrm{O}_{4}-30 \mathrm{rGO}$ nanocomposite.

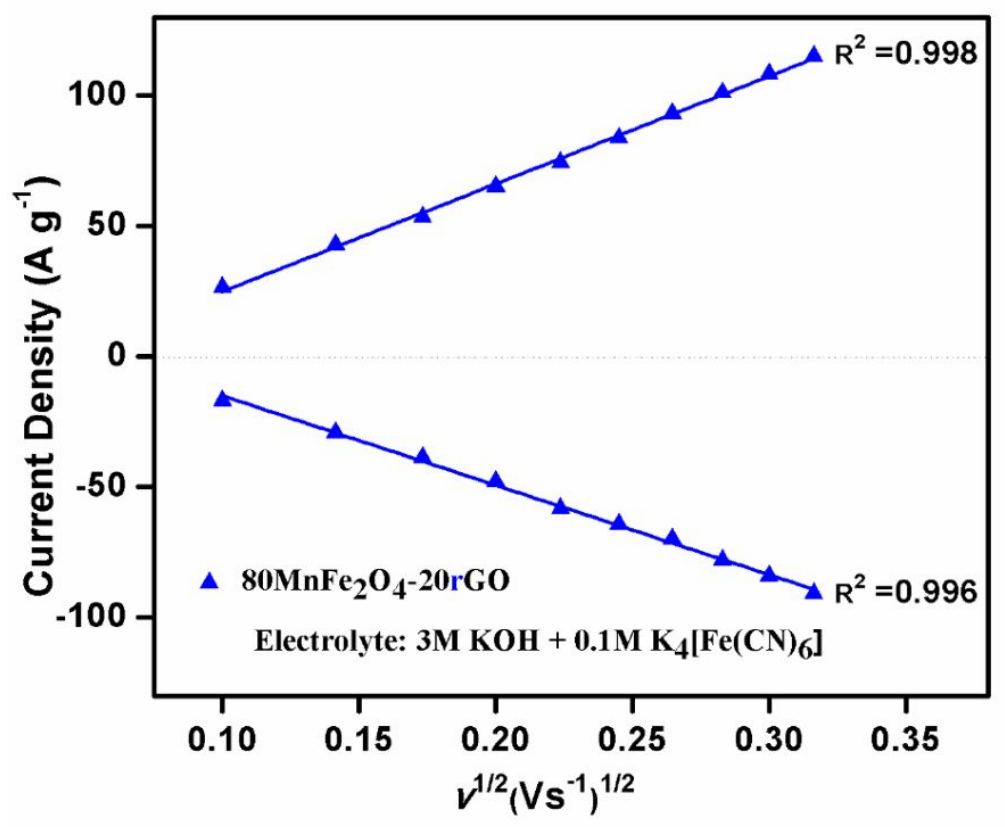

Figure S10. Randles-Sevcik plot of $80 \mathrm{MnFe}_{2} \mathrm{O}_{4}-20 \mathrm{rGO}$ nanocomposite in $3 \mathrm{M} \mathrm{KOH}+0.1 \mathrm{M}$ $\mathrm{K}_{4}\left[\mathrm{Fe}(\mathrm{CN})_{6}\right]$ 


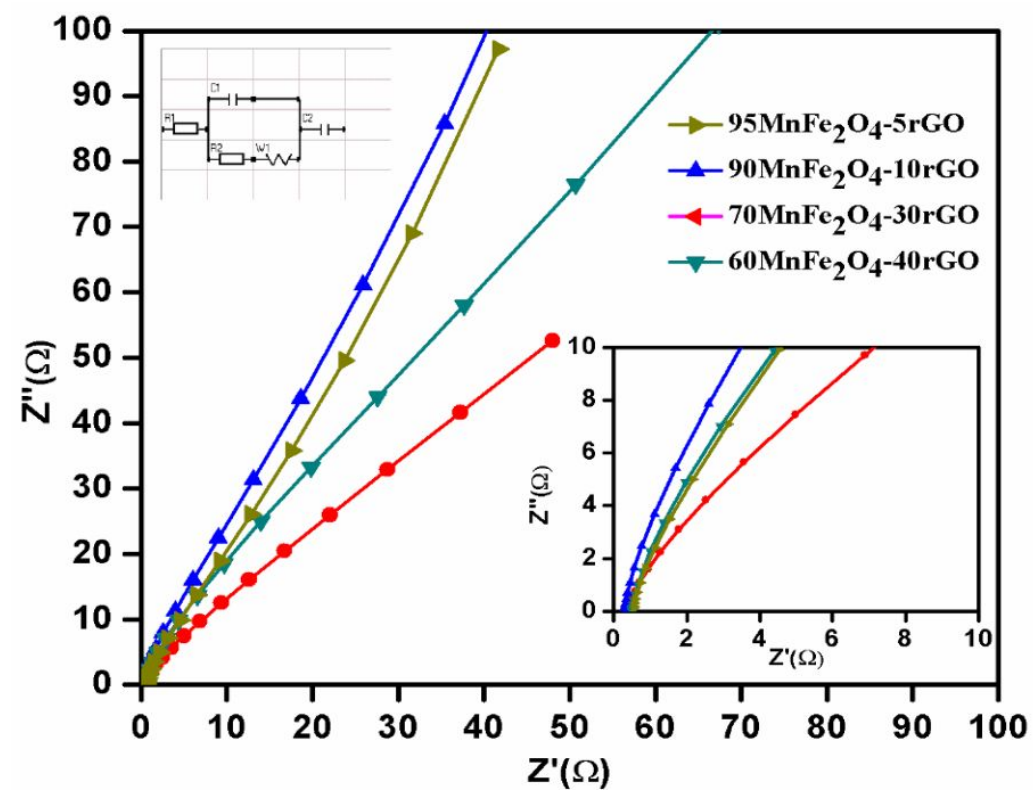

Figure S11. Nyquist plot of $95 \mathrm{MnFe}_{2} \mathrm{O}_{4}-5 \mathrm{rGO}, 90 \mathrm{MnFe}_{2} \mathrm{O}_{4}-10 \mathrm{rGO}, 70 \mathrm{MnFe}_{2} \mathrm{O}_{4}-30 \mathrm{rGO}$, and $\mathrm{MnFe}_{2} \mathrm{O}_{4}-40 \mathrm{rGO}$ electrode in $3 \mathrm{M} \mathrm{KOH}+0.1 \mathrm{M} \mathrm{K}_{4}\left[\mathrm{Fe}(\mathrm{CN})_{6}\right]$ electrolyte. Inset shows the high frequency region of the impedance spectra and equivalent circuit used for fitting the Nyquist plots.

Table S2. Fitting results of the EIS data of all the synthesized nanocomposites.

\begin{tabular}{|c|c|c|c|c|c|c|}
\hline S.No. & Material & $\begin{array}{l}\text { Equivalent } \\
\text { Series } \\
\text { Resistance } \\
\left(\mathbf{R}_{1}=\mathbf{R}_{\mathrm{s}}\right) \\
\Omega\end{array}$ & $\begin{array}{l}\text { Charge } \\
\text { Transfer } \\
\text { Resistance } \\
\left(\mathbf{R}_{2}=\mathbf{R}_{\mathrm{ct}}\right) \mathbf{\Omega}\end{array}$ & $\begin{array}{c}\text { W1 } \\
\text { (Warburg) } \\
\Omega\end{array}$ & $\begin{array}{c}\mathrm{C1}=\mathrm{C}_{\mathrm{DL}} \\
\text { (F) }\end{array}$ & $\begin{array}{c}C 2=C_{P S} \\
\text { (F) }\end{array}$ \\
\hline 1. & Pure $\mathrm{MnFe}_{2} \mathrm{O}_{4}$ & 2.12 & 10.13 & $1.57 \mathrm{E}-02$ & $1.12 \mathrm{E}-03$ & $8.08 \mathrm{E}-02$ \\
\hline 2. & rGO & 2.15 & 2.86 & $1.04 \mathrm{E}-02$ & $6.43 \mathrm{E}-05$ & $2.09 \mathrm{E}-02$ \\
\hline 3. & $95 \mathrm{MnFe}_{2} \mathrm{O}_{4}-5 \mathrm{rGO}$ & 0.50 & 0.45 & $8.06 \mathrm{E}-02$ & $1.34 \mathrm{E}-03$ & $5.07 \mathrm{E}-02$ \\
\hline 4. & $90 \mathrm{MnFe}_{2} \mathrm{O}_{4}-10 \mathrm{rGO}$ & 0.30 & 0.34 & $5.54 \mathrm{E}-03$ & 7.94E-03 & $5.78 \mathrm{E}-03$ \\
\hline 5. & $80 \mathrm{MnFe}_{2} \mathrm{O}_{4}-20 \mathrm{rGO}$ & 0.39 & 0.31 & $1.72 \mathrm{E}-02$ & $1.40 \mathrm{E}-03$ & $1.0 \mathrm{E}-01$ \\
\hline 6. & $70 \mathrm{MnFe}_{2} \mathrm{O}_{4}-30 \mathrm{rGO}$ & 0.53 & 0.65 & $9.85 \mathrm{E}-03$ & $1.30 \mathrm{E}-03$ & $3.32 \mathrm{E}-02$ \\
\hline 7. & $60 \mathrm{MnFe}_{2} \mathrm{O}_{4}-40 \mathrm{rGO}$ & 0.51 & 1.3 & $7.49 \mathrm{E}-03$ & $1.44 \mathrm{E}-03$ & $6.26 \mathrm{E}-01$ \\
\hline
\end{tabular}


Table S3. Comparison of electrochemical performance of Manganese ferrite and graphene-based nanocomposites of two electrode symmetric supercapacitors.

\begin{tabular}{|c|c|c|c|c|c|c|}
\hline S.No. & Electrolyte & $\begin{array}{c}\text { Specific } \\
\text { capacitance } \\
\left(\mathrm{F} \mathrm{g}^{-1}\right)\end{array}$ & $\begin{array}{c}\text { Power } \\
\text { Density } \\
\left(\mathrm{W} \mathrm{kg}^{-1}\right)\end{array}$ & 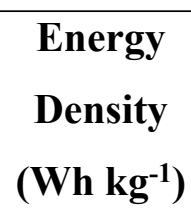 & Retention & Reference \\
\hline 1) & $2 \mathrm{M} \mathrm{LiNO}_{3}$ & - & - & 14.5 & $\begin{array}{c}82 \% \\
\text { (1000cycles) }\end{array}$ & 1 \\
\hline 2) & $6 \mathrm{M} \mathrm{KOH}$ & $\begin{array}{c}152.5 \\
\left(0.1 \mathrm{~A} \mathrm{~g}^{-1}\right)\end{array}$ & - & - & - & 2 \\
\hline 3) & $3.5 \mathrm{M} \mathrm{KOH}$ & $\begin{array}{c}245 \\
\left(0.2 \mathrm{~A} \mathrm{~g}^{-1}\right)\end{array}$ & 120 & 12.6 & $\begin{array}{c}105 \% \\
(10,000 \text { cycles })\end{array}$ & 3 \\
\hline 4) & $1 \mathrm{M} \mathrm{NaCl}$ & $\begin{array}{c}55 \\
\left(200 \mathrm{mV} \mathrm{s}^{-1}\right)\end{array}$ & 11000 & 7.6 & - & 4 \\
\hline 5) & $1 \mathrm{M} \mathrm{H}_{2} \mathrm{SO}_{4}$ & $\begin{array}{c}269 \\
\left(0.5 \mathrm{~A} \mathrm{~g}^{-1}\right)\end{array}$ & 298.6 & 13.3 & $\begin{array}{c}97 \% \\
(10000 \text { cycles })\end{array}$ & 5 \\
\hline 6) & $6 \mathrm{M} \mathrm{KOH}$ & $\begin{array}{c}271.5 \\
\left(0.5 \mathrm{~A} \mathrm{~g}^{-1}\right)\end{array}$ & 324.5 & 15.9 & $\begin{array}{c}104 \% \\
(5000 \text { cycles })\end{array}$ & 6 \\
\hline 7) & $1 \mathrm{M} \mathrm{KOH}$ & $\begin{array}{c}307.2 \\
\left(0.1 \mathrm{~A} \mathrm{~g}^{-1}\right)\end{array}$ & - & 13.5 & $\begin{array}{c}74 \% \\
(2000 \text { cycles })\end{array}$ & 7 \\
\hline 8) & $\mathrm{H}_{2} \mathrm{SO}_{4} / \mathrm{PVA}$ & $\begin{array}{c}120 \\
\left(0.1 \mathrm{~A} \mathrm{~g}^{-1}\right)\end{array}$ & 400 & 5 & $\begin{array}{c}105 \% \\
(5000 \text { cycles })\end{array}$ & 8 \\
\hline 9) & $\begin{array}{l}0.5 \mathrm{M} \\
\mathrm{H}_{2} \mathrm{SO}_{4}\end{array}$ & $\begin{array}{c}205.6 \\
\left(0.5 \mathrm{~A} \mathrm{~g}^{-1}\right)\end{array}$ & - & 16 & $\begin{array}{c}75 \% \\
(100000 \text { cycles })\end{array}$ & 9 \\
\hline 10) & $1 \mathrm{M} \mathrm{NaCl}$ & $\begin{array}{c}51.8 \\
\left(5 \mathrm{mV} \mathrm{s}^{-1}\right)\end{array}$ & 3076 & 10.25 & $\begin{array}{c}95.3 \% \\
(100 \text { cycles })\end{array}$ & 10 \\
\hline 11) & $\begin{array}{c}3 \mathrm{M} \mathrm{KOH}+ \\
0.1 \mathrm{M} \\
\mathrm{K}_{4}\left[\mathrm{Fe}(\mathrm{CN})_{6}\right]\end{array}$ & $\begin{array}{c}468 \\
\left(3 \mathrm{~A} \mathrm{~g}^{-1}\right)\end{array}$ & 1500 & 16.25 & $\begin{array}{c}80 \%(4000 \\
\text { cycles })\end{array}$ & This work \\
\hline
\end{tabular}



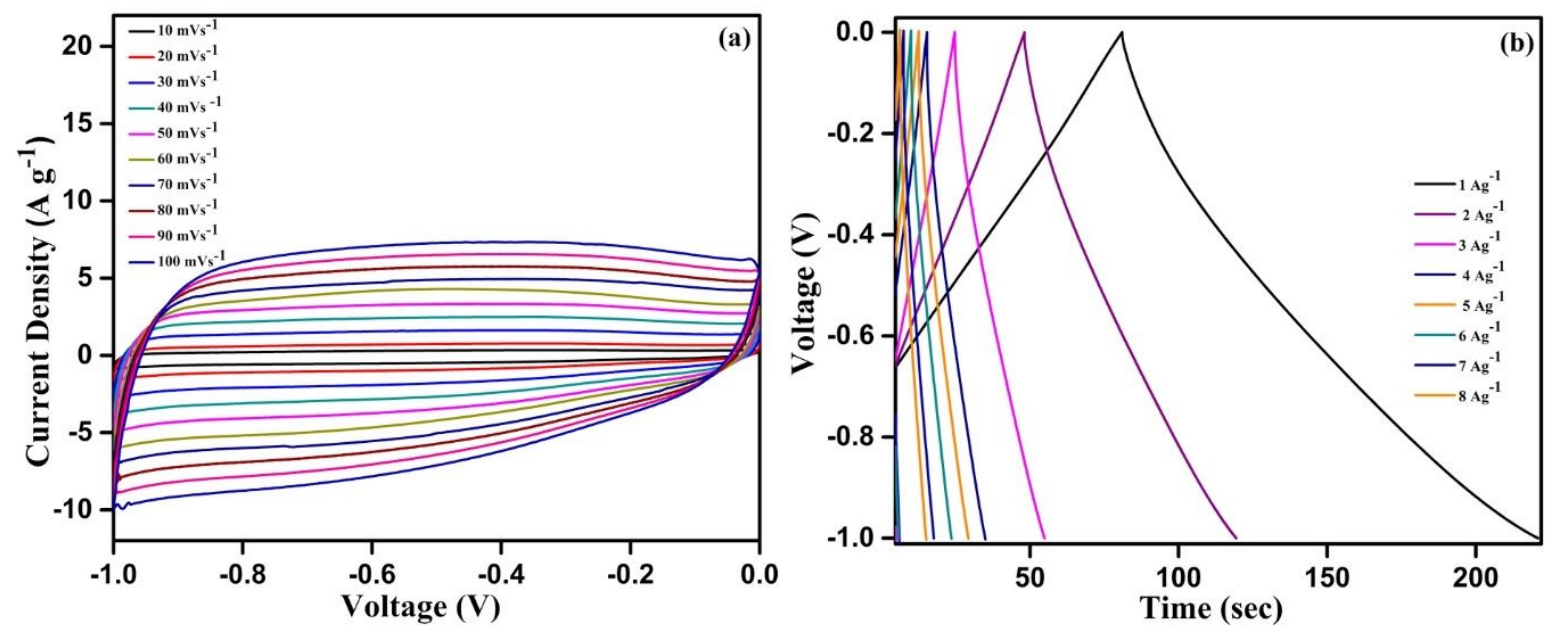

Figure S12. (a) CV curves at different scan rates ranging from 10-100 $\mathrm{mV} \mathrm{s}^{-1}$, (b) GCD curves at different current densities of $\mathrm{rGO}$ as the negative electrode in $3 \mathrm{M} \mathrm{KOH}+0.1 \mathrm{M} \mathrm{K}_{4}\left[\mathrm{Fe}(\mathrm{CN})_{6}\right]$ electrolyte.

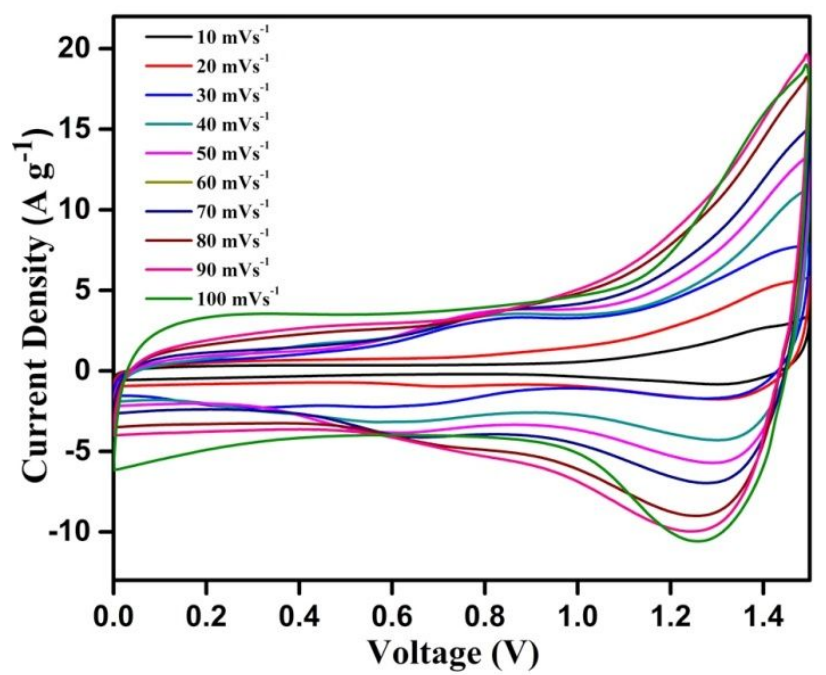

Figure S13. $\mathrm{CV}$ curves of $80 \mathrm{MnFe}_{2} \mathrm{O}_{4}-20 \mathrm{rGO} / / \mathrm{rGO}$ asymmetric electrode in $3 \mathrm{M} \mathrm{KOH}+0.1 \mathrm{M}$ $\mathrm{K}_{4}\left[\mathrm{Fe}(\mathrm{CN})_{6}\right]$ electrolyte at different scan rate ranging from $10-100 \mathrm{mV} \mathrm{s}^{-1}$. 


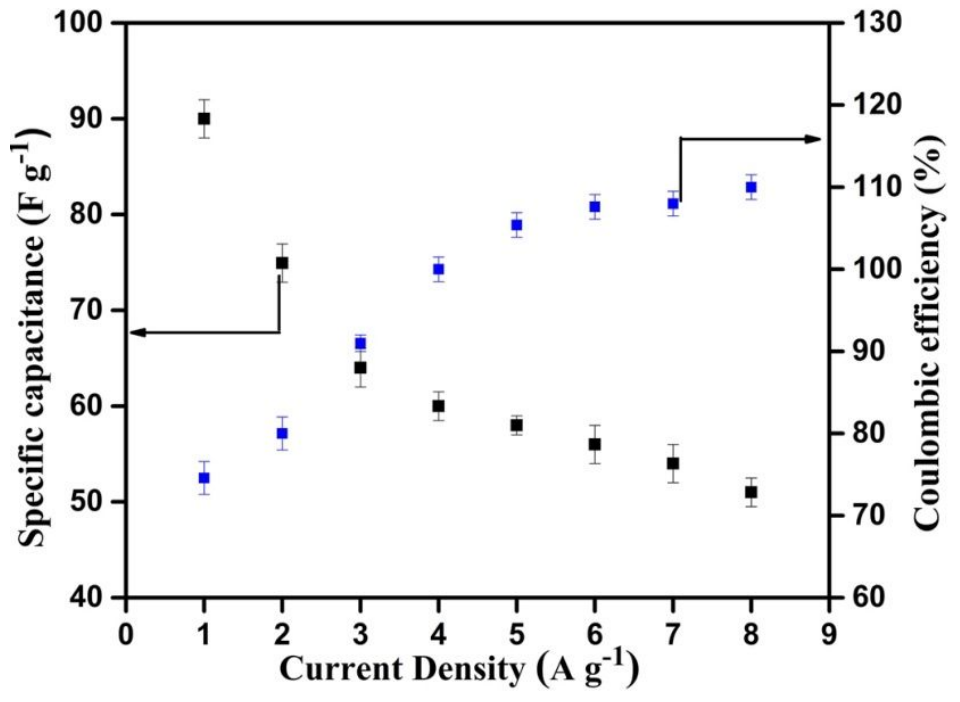

Figure S14. The plot of variation of specific capacitance and Coulombic efficiency with changing current density.
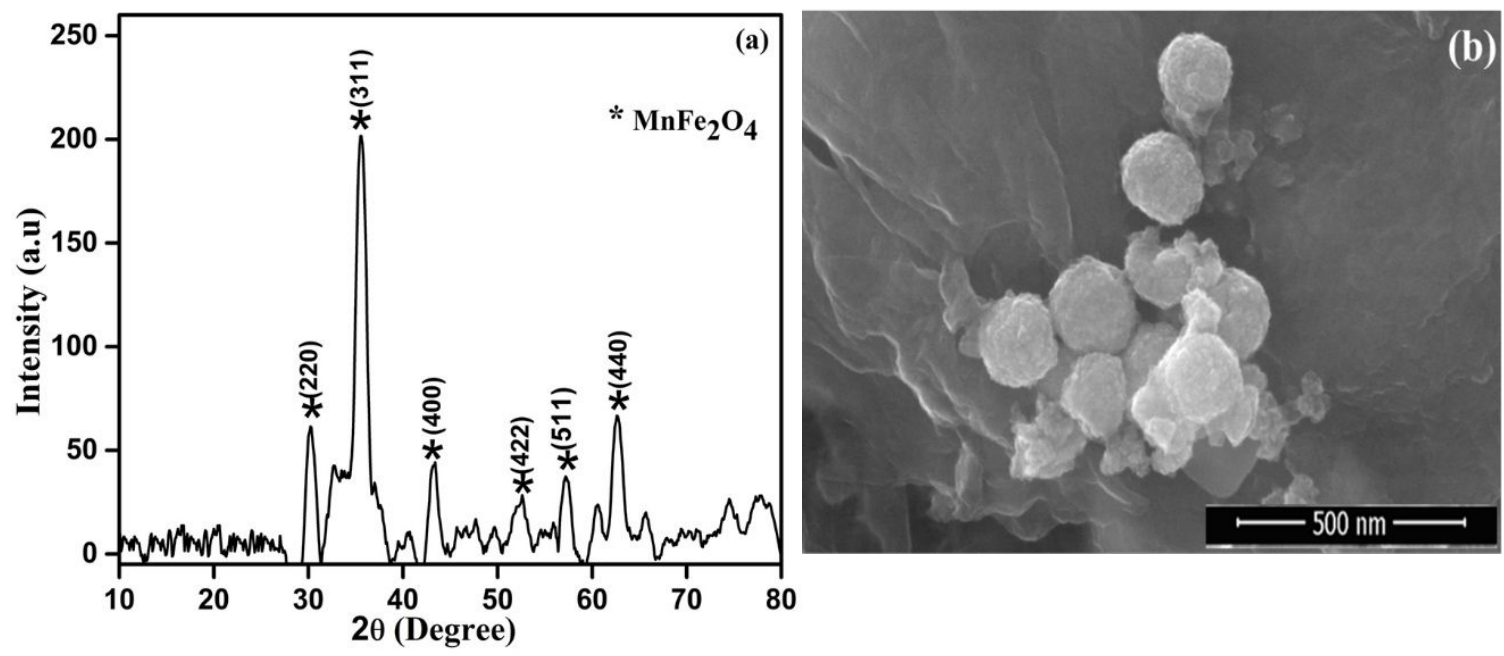

Figure S15. (a) XRD pattern and (b) FESEM micrograph of $80 \mathrm{MnFe}_{2} \mathrm{O}_{4}-20 \mathrm{rGO}$ electrode after chargedischarge cycles. 
Table S4. Comparison of electrochemical performance of some nanocomposites in two-electrode asymmetric supercapacitors.

\begin{tabular}{|c|c|c|c|c|c|c|c|}
\hline S.No. & Material & Electrolyte & $\begin{array}{c}\text { Working } \\
\text { Potential } \\
\text { (V) }\end{array}$ & $\begin{array}{c}\text { Power } \\
\text { Density } \\
\left(\mathbf{W ~ k g}^{-1}\right)\end{array}$ & $\begin{array}{c}\text { Energy } \\
\text { Density } \\
\left(\mathbf{W h ~ k g}^{-1}\right)\end{array}$ & Retention & Reference \\
\hline 1) & $\begin{array}{c}\mathrm{ZnFe}_{2} \mathrm{O}_{4} / / \\
\mathrm{NRG} \\
\text { composite }\end{array}$ & $1 \mathrm{M} \mathrm{KOH}$ & -1 to 0 & 3000 & 6.7 & $\begin{array}{c}84.4 \% \\
\text { (1000cycles) }\end{array}$ & 11 \\
\hline 2) & $\mathrm{CoS} / / \mathrm{AC}$ & $3.5 \mathrm{M} \mathrm{KOH}$ & 0 to 1.8 & 1800 & 5.3 & $\begin{array}{c}92 \% \\
\text { (5000 cycles) }\end{array}$ & 12 \\
\hline 3) & $\begin{array}{l}\mathrm{Mn}_{0.9} \mathrm{CuO}_{0.1} \\
\mathrm{Fe}_{2} \mathrm{O}_{4} / / \mathrm{rGO}\end{array}$ & $1 \mathrm{M} \mathrm{Na}_{2} \mathrm{SO}_{4}$ & -0.4 to 1 & - & - & $\begin{array}{c}95 \% \\
(1000 \text { cycles })\end{array}$ & 13 \\
\hline 4) & $\mathrm{CuO} / / \mathrm{AC}$ & $3 \mathrm{M} \mathrm{KOH}$ & 0 to 1.4 & 700 & 19.7 & $\begin{array}{c}96 \% \\
(3000 \text { cycles })\end{array}$ & 14 \\
\hline 5) & $\mathrm{NiO} / /$ carbon & $6 \mathrm{M} \mathrm{KOH}$ & 0 to 1.3 & - & 10 & $\begin{array}{c}74 \% \\
\text { (1000 cycles) }\end{array}$ & 15 \\
\hline 6) & $\begin{array}{l}\text { Ni-Co } \\
\text { oxide//AC }\end{array}$ & $1 \mathrm{M} \mathrm{KOH}$ & -1 to 0 & 1902.9 & 7.4 & $\begin{array}{c}85 \% \\
\text { (2000 cycles) }\end{array}$ & 16 \\
\hline 7) & $\begin{array}{l}\mathrm{Co}(\mathrm{OH})_{2} \\
\text { nanowires// } \\
\mathrm{AC}\end{array}$ & $6 \mathrm{M} \mathrm{KOH}$ & 0 to 1.6 & 153 & 13.6 & $\begin{array}{c}86.3 \% \\
(5000 \text { cycles })\end{array}$ & 17 \\
\hline 8) & $\begin{array}{l}\mathrm{NaMnO}_{2} / / \\
\mathrm{AC}\end{array}$ & $0.5 \mathrm{M} \mathrm{K}_{2} \mathrm{SO}_{4}$ & 0 to 1.9 & 130 & 19.5 & $\begin{array}{l}10000 \\
\text { cycles }\end{array}$ & 18 \\
\hline 9) & $\begin{array}{l}\mathrm{MnO}_{2} / / \text { Grap } \\
\text { hene } \\
\text { hydrogel }\end{array}$ & $\begin{array}{c}0.1 \mathrm{M} \\
\mathrm{Na}_{2} \mathrm{SO}_{4}\end{array}$ & 0 to 2 & 1000 & 23.2 & $\begin{array}{c}83.4 \% \\
\text { (5000 cycles) }\end{array}$ & 19 \\
\hline 10) & $\begin{array}{l}\text { Carbon } \\
\text { spheres/ } \\
\mathrm{MnO}_{2} / /\end{array}$ & $\begin{array}{c}0.1 \mathrm{M} \\
\mathrm{Na}_{2} \mathrm{SO}_{4}\end{array}$ & 0 to 2 & 100 & 22.1 & $\begin{array}{c}99 \% \\
\text { (1000 cycles) }\end{array}$ & 20 \\
\hline
\end{tabular}




\begin{tabular}{|c|c|c|c|c|c|c|c|}
\hline & $\begin{array}{l}\text { carbon } \\
\text { spheres }\end{array}$ & & & & & & \\
\hline 11) & $\begin{array}{l}\mathrm{Co}_{3} \mathrm{O}_{4} \text { NSs- } \\
\mathrm{rGO} / / \mathrm{AC}\end{array}$ & $2 \mathrm{M} \mathrm{KOH}$ & 0 to 1.45 & 2166 & 13.4 & $\begin{array}{c}89 \% \\
(1000 \text { cycles })\end{array}$ & 21 \\
\hline 12) & $\begin{array}{l}\mathrm{NiCo}_{2} \mathrm{O}_{4} \\
\mathrm{NSs} @ \mathrm{HMR} \\
\mathrm{As} / / \mathrm{AC}\end{array}$ & $1 \mathrm{M} \mathrm{KOH}$ & 0 to 1.5 & 7800 & 15.42 & $\begin{array}{c}106 \% \\
(2500 \text { cycles })\end{array}$ & 22 \\
\hline 13) & $\begin{array}{l}\mathrm{Co}_{3} \mathrm{O}_{4} @ \mathrm{Mn} \\
\mathrm{O}_{2} / / \mathrm{MEGO}\end{array}$ & $1 \mathrm{M} \mathrm{LiOH}$ & 0 to 1.6 & 158000 & 17.7 & $\begin{array}{c}95 \% \\
\text { (5000 cycles) }\end{array}$ & 23 \\
\hline 14) & $\begin{array}{l}\mathrm{MnCo}_{2} \mathrm{CO}_{4} / / \\
\mathrm{AC}\end{array}$ & $1 \mathrm{M} \mathrm{LiOH}$ & 0 to 1.6 & 1551 & 19 & $\begin{array}{c}98 \% \\
(3000 \text { cycles })\end{array}$ & 24 \\
\hline 15) & $\begin{array}{l}\mathrm{MnFe}_{2} \mathrm{O}_{4} / / \\
\text { Graphene }\end{array}$ & $1 \mathrm{M} \mathrm{Na}_{2} \mathrm{SO}_{4}$ & 0 to 1.8 & 225 & 25.9 & $\begin{array}{c}90 \% \\
(4000 \text { cycles })\end{array}$ & 25 \\
\hline 16) & $\begin{array}{l}\mathrm{LiMn}_{2} \mathrm{O}_{4} / / \\
\mathrm{MnFe}_{2} \mathrm{O}_{4}\end{array}$ & $\mathrm{LiNO}_{3}$ & 0 to 1.8 & 1800 & 5.5 & $\begin{array}{c}90 \% \\
\text { (5000 cycles) }\end{array}$ & 26 \\
\hline 17) & $\begin{array}{c}\mathrm{MnFe}_{2} \mathrm{O}_{4^{-}} \\
\mathrm{rGO} / / \\
\mathrm{rGO}\end{array}$ & $\begin{array}{c}3 \mathrm{M} \mathrm{KOH}+ \\
0.1 \mathrm{M} \\
\mathrm{K}_{4}\left[\mathrm{Fe}(\mathrm{CN})_{6}\right]\end{array}$ & 0 to 1.5 & 750 & 28 & $\begin{array}{c}95 \% \\
(4000 \text { cycles })\end{array}$ & This work \\
\hline
\end{tabular}




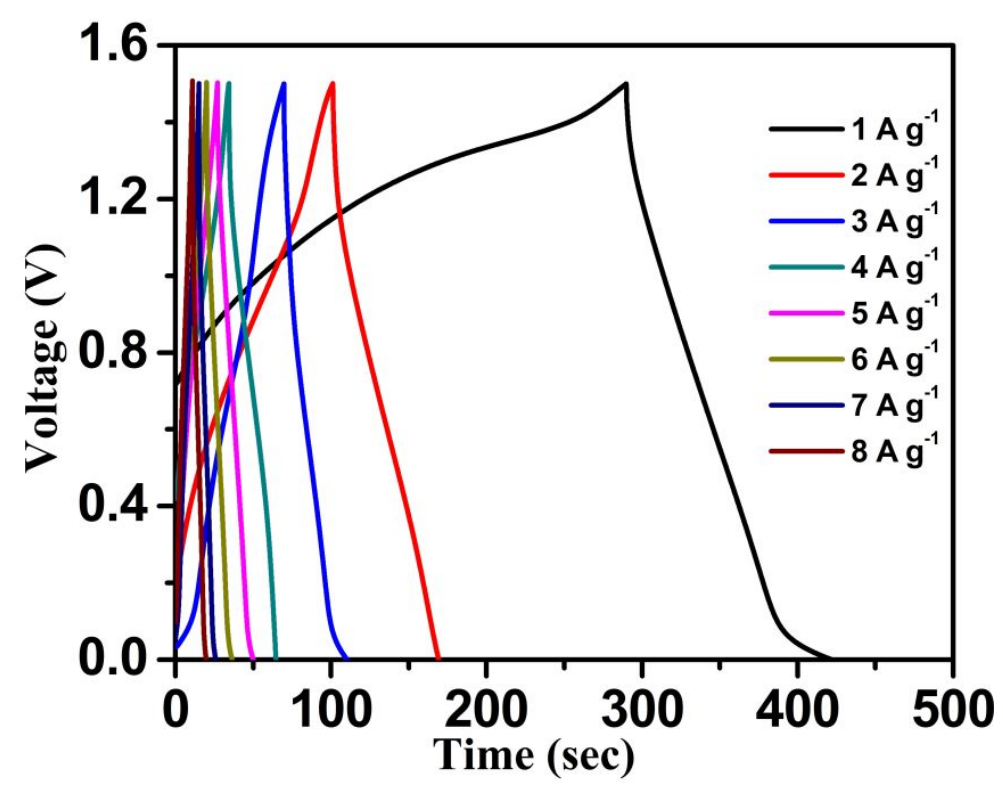

Figure S16. GCD curves of $80 \mathrm{MnFe}_{2} \mathrm{O}_{4}-20 \mathrm{rGO} / / \mathrm{rGO}$ all-solid-state flexible asymmetric device at different current densities $\left(1-8 \mathrm{~A} \mathrm{~g}^{-1}\right)$ in $3 \mathrm{M} \mathrm{KOH}+0.1 \mathrm{M} \mathrm{K}_{4}\left[\mathrm{Fe}(\mathrm{CN})_{6}\right]$.

\section{REFERENCES}

1. Wang, B.; Guo, P.; Bi, H.; Li, Q.; Zhang, G.; Wang, R.; Liu, J.; Zhao, X. Electrocapacitive properties of $\mathrm{MnFe}_{2} \mathrm{O}_{4}$ electrodes in aqueous $\mathrm{LiNO}_{3}$ electrolyte with surfactants. Int. J. Electrochem. Sci. 2013, 8, 8966-8977.

2. Wang, R.; Li, Q.; Cheng, L.; Li, H.; Wang, B.; Zhao, X.; Guo, P. Electrochemical properties of manganese ferrite-based supercapacitors in aqueous electrolyte: the effect of ionic radius. Colloids Surf. A: Physicochemical and Engineering Aspects. 2014, 457, 94-99.

3. Vignesh, V.; Subramani, K.; Sathish, M.; Navamathavan, R. Electrochemical investigation of manganese ferrites prepared via a facile synthesis route for supercapacitor applications. Colloids Surf. A: Physicochemical and Engineering Aspects. 2018, 538, 668-677.

4. Kuo, S.-L.; Wu, N.-L. Electrochemical characterization on $\mathrm{MnFe}_{2} \mathrm{O}_{4} /$ carbon black composite aqueous supercapacitors. J. Power Sources. 2006, 162, 1437-1443. 
5. Ghadimi, L. S.; Arsalani, N.; Tabrizi, A. G.; Mohammadi, A.; Ahadzadeh, I., Novel nanocomposite of $\mathrm{MnFe}_{2} \mathrm{O}_{4}$ and nitrogen-doped carbon from polyaniline carbonization as electrode material for symmetric ultra-stable supercapacitor. Electrochim. Acta. 2018, 282, 116-127.

6. Tabrizi, A. G.; Arsalani, N.; Mohammadi, A.; Namazi, H.; Ghadimi, L. S.; Ahadzadeh, I. Facile synthesis of a $\mathrm{MnFe}_{2} \mathrm{O}_{4} / \mathrm{rGO}$ nanocomposite for an ultra-stable symmetric supercapacitor. New $J$. Chem. 2017, 41, 4974-4984.

7. Xiong, P.; Hu, C.; Fan, Y.; Zhang, W.; Zhu, J.; Wang, X. Ternary manganese ferrite/graphene/polyaniline nanostructure with enhanced electrochemical capacitance performance. $J$. Power Sources. 2014, 266, 384-392.

8. Cai, W.; Lai, T.; Dai, W.; Ye, J. A facile approach to fabricate flexible all-solid-state supercapacitors based on $\mathrm{MnFe}_{2} \mathrm{O}_{4}$ /graphene hybrids. J. Power Sources. 2014, 255, 170-178.

9. Zha, D.; Xiong, P.; Wang, X. Strongly coupled manganese ferrite/carbon black/polyaniline hybrid for low-cost supercapacitors with high rate capability. Electrochim. Acta. 2015, 185, 218-228. 10. Sankar, K. V.; Selvan, R. K. The preparation of $\mathrm{MnFe}_{2} \mathrm{O}_{4}$ decorated flexible graphene wrapped with PANI and its electrochemical performances for hybrid supercapacitors. $R S C A d v . \mathbf{2 0 1 4}, 4,17555$ 17566.

11. Li, L.; Bi, H.; Gai, S.; He, F.; Gao, P.; Dai, Y.; Zhang, X.; Yang, D.; Zhang, M.; Yang, P. Uniformly dispersed $\mathrm{ZnFe}_{2} \mathrm{O}_{4}$ nanoparticles on nitrogen-modified graphene for high-performance supercapacitor as electrode. Sci. Rep. 2017, 7, 43116.

12. Subramani, K.; Sudhan, N.; Divya, R.; Sathish, M. All-solid-state asymmetric supercapacitors based on cobalt hexacyanoferrate-derived $\mathrm{CoS}$ and activated carbon. $R S C A d v$. 2017, 7, 6648-6659. 
13. Bashir, B.; Shaheen, W.; Asghar, M.; Warsi, M. F.; Khan, M. A.; Haider, S.; Shakir, I.; Shahid, M. Copper doped manganese ferrites nanoparticles anchored on graphene nano-sheets for high performance energy storage applications. J. Alloys Compd. 2017, 695, 881-887.

14. Moosavifard, S. E.; El-Kady, M. F.; Rahmanifar, M. S.; Kaner, R. B.; Mousavi, M. F. Designing 3D highly ordered nanoporous $\mathrm{CuO}$ electrodes for high-performance asymmetric supercapacitors. ACS Appl. Mater. Interfaces. 2015, 7, 4851-4860.

15. Wang, D.-W.; Li, F.; Cheng, H.-M. Hierarchical porous nickel oxide and carbon as electrode materials for asymmetric supercapacitor. J. Power Sources. 2008, 185, 1563-1568.

16. Tang, C.; Tang, Z.; Gong, H. Hierarchically porous Ni-Co oxide for high reversibility asymmetric full-cell supercapacitors. J. Electrochem. Soc. 2012, 159, A651-A656.

17. Tang, Y.; Liu, Y.; Yu, S.; Mu, S.; Xiao, S.; Zhao, Y.; Gao, F. Morphology controlled synthesis of monodisperse cobalt hydroxide for supercapacitor with high performance and long cycle life. $J$. Power Sources. 2014, 256, 160-169.

18. Qu, Q.; Li, L.; Tian, S.; Guo, W.; Wu, Y.; Holze, R. A cheap asymmetric supercapacitor with high energy at high power: activated carbon $/ / \mathrm{K}_{0.27} \mathrm{MnO}_{2} .0 .6 \mathrm{H}_{2} \mathrm{O}$. J. Power Sources. 2010, 195, 27892794.

19. Gao, H.; Xiao, F.; Ching, C. B.; Duan, H. High-performance asymmetric supercapacitor based on graphene hydrogel and nanostructured $\mathrm{MnO}_{2}$. ACS App. Mater Interfaces. 2012, 4, 2801-2810.

20. Lei, Z.; Zhang, J.; Zhao, X. Ultrathin $\mathrm{MnO}_{2}$ nanofibers grown on graphitic carbon spheres as high-performance asymmetric supercapacitor electrodes. J. Mater. Chem. 2012, 22, 153-160.

21. Yuan, C.; Zhang, L.; Hou, L.; Pang, G.; Oh, W.-C. One-step hydrothermal fabrication of strongly coupled $\mathrm{Co}_{3} \mathrm{O}_{4}$ nanosheets-reduced graphene oxide for electrochemical capacitors. $R S C$ Adv. 2014, 4, 14408-14413. 
22. Lu, X.-F.; Wu, D.-J.; Li, R.-Z.; Li, Q.; Ye, S.-H.; Tong, Y.-X.; Li, G.-R. Hierarchical $\mathrm{NiCo}_{2} \mathrm{O}_{4}$ nanosheets@ hollow microrod arrays for high-performance asymmetric supercapacitors. J. Mater.

Chem. A 2014, 13, 4706-4713

23. Huang, M.; Zhang, Y.; Li, F.; Zhang, L.; Wen, Z.; Liu, Q. Facile synthesis of hierarchical $\mathrm{Co}_{3} \mathrm{O}_{4} @ \mathrm{MnO}_{2}$ core-shell arrays on $\mathrm{Ni}$ foam for asymmetric supercapacitors. J. Power Sources. 2014, 252, 98-106.

24. Krishnan, S. G.; Harilal, M.; Pal, B.; Misnon, I. I.; Karuppiah, C.; Yang, C.-C.; Jose, R. Improving the symmetry of asymmetric supercapacitors using battery-type positive electrodes and activated carbon negative electrodes by mass and charge balance. J. Electroanal. Chem. 2017, 805, 126-132.

25. Li, B.; Fu, Y.; Xia, H.; Wang, X. High-performance asymmetric supercapacitors based on $\mathrm{MnFe}_{2} \mathrm{O}_{4}$ /graphene nanocomposite as anode material. Mater. Lett. 2014, 122, 193-196.

26. Lin, Y.-P.; Wu, N.-L. Characterization of $\mathrm{MnFe}_{2} \mathrm{O}_{4} / \mathrm{LiMn}_{2} \mathrm{O}_{4}$ aqueous asymmetric supercapacitor. J. Power Sources. 2011, 196, 851-854. 